\title{
The lingering manifestations of COVID-19 \\ during and after convalescence: update on long-term pulmonary consequences of coronavirus disease 2019 (COVID-19)
}

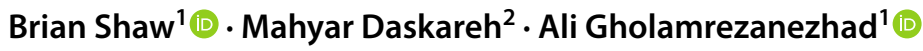

Received: 14 June 2020 / Accepted: 21 September 2020 / Published online: 1 October 2020

(C) Italian Society of Medical Radiology 2020

\begin{abstract}
The long-term sequelae of coronavirus disease 2019 (COVID-19) are still unknown. Lessons from past viral epidemics reveal that, after recovery, patients with viral pulmonary infections can suffer from irreversible pulmonary dysfunction and demonstrate residual imaging or functional abnormalities. Residual ground glass opacities, consolidations, reticular and linear opacities, residual crazy paving pattern, melted sugar sign, and parenchymal fibrotic bands are several features found in the late or remission stages of COVID-19. These radiologic findings have been observed weeks after symptom onset, even after hospital discharge, and they may or may not correlate with clinical manifestations. High-resolution CT may be indicated to establish new baselines and track changes in residual impairments. In our previous review, we observed significant pulmonary sequelae in some COVID-19 survivors at follow-up. In this update, we review the current literature on the clinical and radiologic manifestations of post-recovery COVID-19 toward the end of hospital admission and after discharge.
\end{abstract}

Keywords COVID-19 $\cdot$ High-resolution CT $\cdot$ SARS-CoV-2 $\cdot$ Co-morbidities $\cdot$ Surveillance $\cdot$ Recovery

\section{Introduction}

In December 2019, a severe respiratory illness outbreak appeared in Wuhan, China. The causative agent was identified as a novel coronavirus, later termed Severe Acute Respiratory Syndrome-Coronavirus-2 (SARS-CoV-2) by the World Health Organization (WHO). Infection by SARS-CoV-2, termed Coronavirus disease 2019 (COVID19), causes most commonly fever, cough, fatigue, and dyspnea [1-5]. In severe cases, COVID-19 results in severe acute respiratory syndrome, respiratory failure, and death. Multiple different extrapulmonary findings and manifestations have been also described in this disease. SARS-CoV-2 is a

Ali Gholamrezanezhad

ali.gholamrezanezhad@med.usc.edu

Brian Shaw

briansha@usc.edu; bshaw006@gmail.com

Mahyar Daskareh

daskarehmahyar@gmail.com

1 Keck School of Medicine, University of Southern California, Los Angeles, CA 90033, USA

2 Independent Researcher, Tehran, Iran highly contagious virus. Given the alarming number of cases and deaths, the WHO declared COVID-19 as a pandemic in early March 2020 [1].

Over the past several months, the risk factors, clinical manifestations, diagnosis, and management of COVID-19 have been investigated in numerous publications. Older age, male sex, diabetes mellitus, hypertension, and cardiovascular diseases are all associated with higher rates of respiratory failure, ICU admission, and mortality [2-4]. Although there is published literature describing the clinical and radiologic progression and course of COVID-19 throughout the hospital stay, there is a scarce of literature describing how that progression should guide management at discharge and postdischarge surveillance. In our previous review, we noted that past epidemics including the first SARS and avian flu H7N9 can result in permanent pulmonary damage, observable on chest CT many years after the acute pneumonia. We observed significant pulmonary sequelae in some COVID19 survivors at follow-up as well [5-7]. The purpose of this update is to review the literature in the past several months on the clinical and radiologic features of COVID-19 toward the end of hospital admission and after discharge. 


\section{Clinical findings of COVID-19 at and after discharge}

Most studies clinically stage COVID-19 infection from the onset of symptoms. The number, duration, nomenclature, and discharge criteria for these stages are highly variable across multiple studies. In a rapid advice guideline, Jin et al. find that COVID-19 can be staged anywhere from two to seven stages, depending on the management perspective. In the most general sense, COVID-19 progression can be categorized simply as early or late stage. On the other hand, the progression can be staged more granularly (ultra-early, early, rapid progression, consolidation, and dissipation) [8]. This variability arises from the diversity in clinical presentation and other factors. The discharge criteria in many of these studies are unclear, and few have reported short-term (1-2 months) follow-ups on COVID-19 patients after hospital discharge.

While not mentioned in many studies from China, standardized discharge criteria for COVID-19 have been recommended by the China National Health Commission [9]. As noted by Zheng et al., studies have commonly used the following discharge criteria: (1) two consecutive negative RT-PCR tests; (2) improvements in chest radiography or chest CT; (3) improvement in clinical symptoms, vitals, and laboratory studies; and (4) corticosteroids completely discontinued for more than 1 week with the other criteria met. However, these criteria are not uniform across studies. For instance, the interval time between negative RTPCR tests can be between 24 and $96 \mathrm{~h}$ or longer. Improvement in imaging findings may include reduction in the number and size or resolution of GGOs, decrease in the consolidation density, melted sugar sign (gradual reduction in the density of pulmonary consolidation and transition to ground glass opacity), and residual fibrous stripes/ fibrotic bands. Clinical improvement may be defined as (1) normal body temperature for three to seven days or longer; (2) normal blood oxygen saturation without supplemental oxygen; (3) normal laboratory studies; and (4) symptomatic improvement [10]. These criteria do not include pulmonary function testing or spirometry. Notably, Mo et al. find that, while meeting the criteria at discharge, patients demonstrated reduced carbon monoxide diffusion capacity (DLCO) and predicted total lung capacity (TLC). Patients in the severe pneumonia group had significantly reduced DLCO and TLC compared to both the mild illness and pneumonia groups. Furthermore, the blood oxygen saturation was not different across the mild illness, pneumonia, and severe pneumonia groups [11].

Despite these discharge criteria, studies have shown laboratory and clinical abnormalities, persistent and/or relapsing. Cases of RT-PCR positivity after hospital discharge have been reported $[9,10,12]$. RT-PCR testing of the lower respiratory tract may show positivity for up to two weeks, while testing urine or stool may show positivity for up to four weeks. Other laboratory abnormalities include progressive lymphopenia and progressive neutrophilia. Relapsing clinical features include fever, cough, fatigue, dyspnea, and shortness of breath [10]. In Xing $\mathrm{Y}$ et al., two medical staff who contracted and recovered from COVID-19 infection underwent multiple repeat RTPCR testing for up to 12 days post-discharge. Although both cases reported no symptoms, RT-PCR showed weakly positive in two instances and positive in one instance for each case [12]. In Dou et al., a father and his daughter both hospitalized with COVID-19 were both discharged on February 9 . Both met the criteria of two consecutive negative RT-PCR tests with at least $24 \mathrm{~h}$ interval at discharge. The father and daughter were in isolation at home for 17 days until February 26 when they each received an additional RT-PCR test and HRCT. Both follow-up RT-PCR tests were positive. While significantly improved from the last CTs of their hospital admissions, both follow-up CT scans showed persistent abnormalities. The study notes no evidence of reinfection or person-to-person transmission [9]. $\mathrm{In} \mathrm{Fu} \mathrm{W}$ et al., three patients previously discharged tested positive on subsequent RT-PCR, prompting readmission although asymptomatic. The times from the first discharge to second readmission were 7,12, and 9 days. During readmission, the repeat HRCT of case 2 showed residual airspace opacity. Repeat HRCTs of case 1 and 3 showed no residual pulmonary opacity. Repeat antibody testing showed negative $\operatorname{IgM}$ and positive $\operatorname{IgG}$ for all patients. At the second discharge, they again had two consecutive negative RT-PCR tests, were discharged, and were encouraged to isolate for 14 days in a hotel [13].

Histologically, Yao et al. illustrate that even in a COVID19 patient anticipating discharge, there is pathological evidence for residual abnormalities after recovery. In their case report, an elderly COVID-19 patient met criteria and expected discharge when she suddenly experienced a fatal cardiac arrest. Although nasopharyngeal RT-PCR testing was negative, further testing on lung sections showed clear viral particles in bronchiolar epithelial cells and type II alveolar epithelial (AE) cells. Biopsy demonstrated diffuse alveolar damage, desquamation of type II AE cells, fibrin exudation, hyaline membranes, scattered interstitial inflammation, monocytes, macrophages, and chronic respiratory disease-associated changes. Yao XH noted that these abnormalities are similar to findings in patients with SARS and MERS [14]. 


\section{Radiologic findings of COVID-19 at and after discharge}

Studies on radiologic progression of severe COVID-19 infection categorize the timeline of imaging findings anywhere from two to six stages with highly variable interval times and number of repeat CT scans. Pan et al. found that patients who had one interval CT scan after 3-14 days demonstrated significant changes in imaging manifestation. The findings include worsening GGOs (multifocal or solitary) or consolidations and development of fibrotic bands, organizing pneumonia, and solid nodules. Imaging signs of improvement included shrinking opacities and nodules and melted sugar signs [15]. Although Chen et al. do not divide the radiologic progression of COVID-19 into stages, patients who underwent repeat imaging showed median times of 7 and 14 days after admission for worsening and improvement, respectively, on CT. All patients underwent chest radiograph or chest $\mathrm{CT}$ on admission [2]. Shi et al. divide COVID-19 infection into four stages by weeks after symptom onset (group $1=$ subclinical, group $2=$ first week, group $3=$ second week, group $4=$ third week). Group 4 showed GGOs, reticular patterns, bronchiolectasis, pleural thickening, and pleural effusions [16].

In a retrospective study, Liang et al. characterized CT findings semiquantitatively by assigning a severity score based on abnormalities occupying percentages of lung lobes. All cases for this study were classified as mild COVID-19 pneumonia per the WHO definition. Patients were categorized into five groups over time: group 1 (days $0-3$ ), group 2 (days 4-7), group 3 (days $8-10$ ), group 4 (days 11-14), and group 5 (days 15-21). There was a significant decreasing trend in patients with both GGOs and consolidation over 21 days, but there was a significant increasing trend in patients with GGOs, consolidation, and linear opacities. However, total lung CT scores were not statistically significant over time, although abnormalities tended to involve more lobes and become bilateral within the first 2 weeks of disease onset. Only 5 patients were known to have been discharged from the hospital [17].

In a similar study, Ding et al. used the same semiquantitative severity score, but found different results. This study divided patients into 6 stages: stage 1 ( $0-4$ days), stage 2 (5-9 days), stage 3 (10-14 days), stage 4 (15-21 days), stage 5 (22-28 days), and stage 6 ( $>28$ days). Contrary to the previous study, differences in CT scores were significant over time, but only in certain lobes. Lower lobes of the lungs were more frequently involved, with significantly higher CT scores at any stage except stage 1. Lesions gradually spread from the periphery to the center of the lungs. Frequency of GGOs, consolidation, crazy-paving pattern, and linear opacities tended to decrease over time.
However, at stage $6,98.1 \%$ of all CT scans still showed abnormalities [18].

Pan et al. take the semiquantitative severity scores further by fitting a curve to the scores of hospitalized COVID-19 patients over time. Severe cases were excluded. Patients were staged as early stage (0-4 days), progressive stage (5-8 days), peak stage (9-13 days), and absorption stage ( $\geq 14$ days) based on CT score quartiles. Peak lung involvement was at 10 days with a CT score of 6 with improvement after 14 days. The bilateral lower lobes scored significantly higher versus the all other lobes and in the peak and absorption stages. During the absorption stage, GGOs and consolidations were observed to absorb. However, based on the fitted curve on total CT scores, the absorption stage extended past 26 days (the last day of follow-up for one patient) [19].

Despite the diversity in staging COVID-19 clinically and radiologically, Jin et al. created a rapid advice guideline based on the current literature. Based on CT imaging, they categorize COVID-19 infection into 5 stages, the last termed a "dissipation stage." During the dissipation stage (roughly 2-3 weeks after symptom onset), the range of lesions reduced, but did not completely resolve. Characteristic findings included patchy consolidation, interlobular septal thickening, and thickening of bronchial walls. The criteria for discharge include (1) body temperature within normal for $\geq 3$ days, (2) improved respiratory symptoms, (3) obvious signs of absorption in the lungs, (4) two consecutive negative RT-PCR tests with at least 1 day sampling interval, and (5) ability to discontinue isolation. This rapid advice guideline does not provide recommendations on follow-up after discharge [8].

\section{Possibility of long-term pulmonary abnormalities}

The literature reports some consistency in the clinical and radiological status of COVID-19 patients at and after discharge. In general, most studies use the same four discharge criteria, but there are inconsistencies in the interval timing of repeat RT-PCR testing and the duration of normal clinical plus laboratory values [10]. Multiple studies find 10 days as the peak of clinical and radiological severity as well as 14 days as a common point for beginning improvement. As mentioned above, several studies use the same chest CT severity scoring system, but only one fits a curve to the data to predict the possible long-term duration of radiological findings [19]. Up to $98.1 \%$ of all chest CTs will show abnormalities at $>28$ days since symptom onset [18], and many case reports with post-discharge surveillance chest $\mathrm{CTs}$ still show abnormalities even though improved $[9,13]$. Clinical and radiological staging is still variable across many studies. Considering the radiological 
data, imaging abnormalities are highly likely to persist in recovered COVID-19 patients. Our limited observations of new survivors are consistent with those in other studies (Figs. 1 and 2). How long these imaging abnormalities persist has yet to be investigated, but these data support long-term pulmonary consequences that we speculated in previous reviews [5-7].
Although the severity of CT findings do not always correlate with severity of clinical manifestations [20,21], there is evidence that COVID-19 is far from over at hospital discharge. However, it is still unclear whether patients are still contagious and whether lung damage is still occurring. For instance, given positive IgG levels after discharge [13], where patients fall in the timeline of humoral response to
Fig. 1 A 64-year-old woman presented with myalgia and low-grade fever to the emergency department. Baseline CT examination showed multifocal patchy ground glass opacities and interlobular septal thickening $(\mathbf{a}, \mathbf{b})$. The diagnosis of COVID-19 was confirmed later by RT-PCR. Follow-up imaging 35 days after initial CT demonstrated residual pure ground glass opacity without focal pleural thickening $(\mathbf{c}, \mathbf{d})$
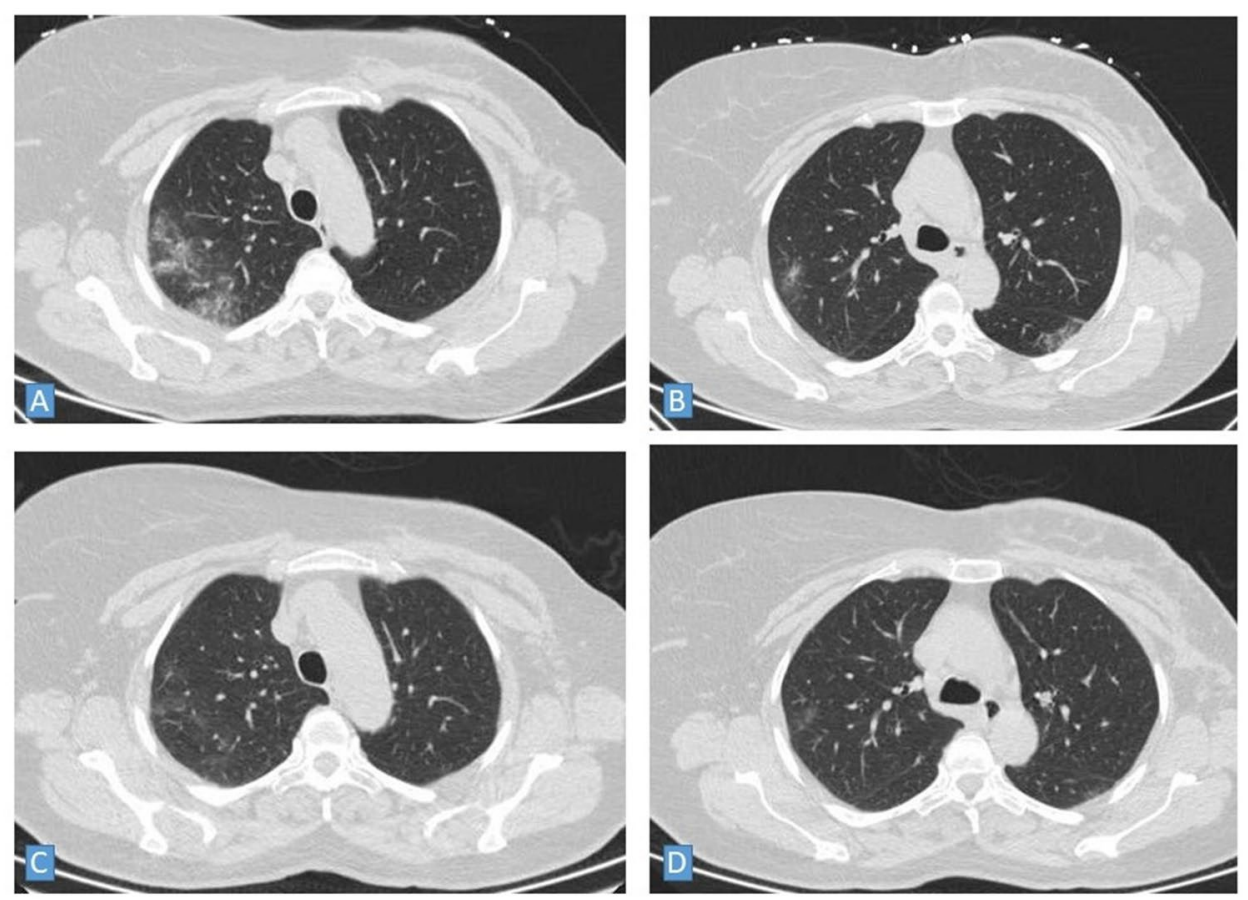
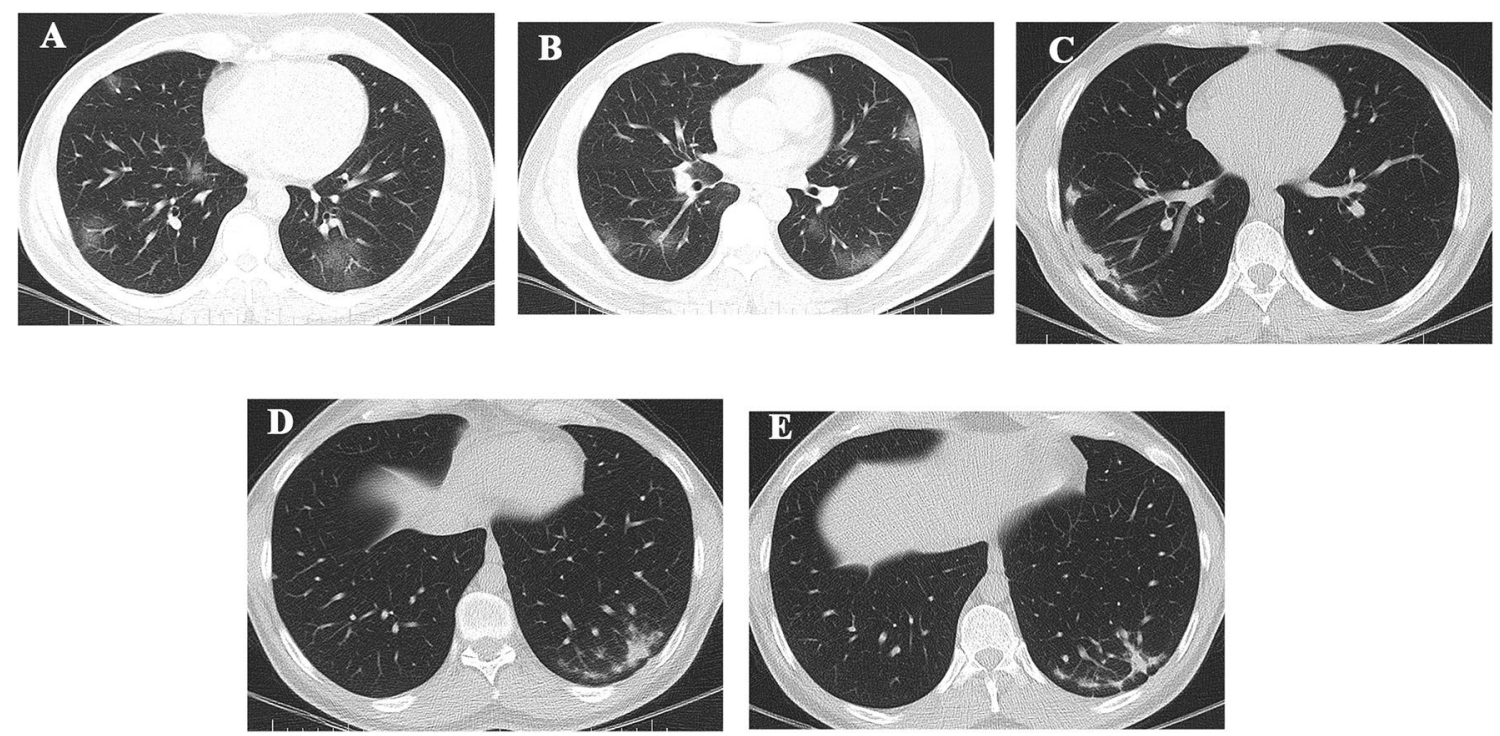

Fig. 2 A 44-year-old man with shortness of breath, cough and fever was referred to us for non-contrast chest CT. Multifocal bilateral peripheral ground glass opacities were identified $(\mathbf{a}, \mathbf{b})$, consistent with COVID-19. He returned for follow-up imaging 34 days after the initial presentation. Follow-up non-contrast chest CT demonstrates residual pulmonary opacities and fibrotic bands involving peripheral bilateral lung bases (c-e) 
SARS-CoV-2 remains unknown. On pathology, the observed signs of chronic lung damage and presence of SARS-CoV-2 particles at discharge suggest long-term pulmonary consequences. For example, these findings were observed in one case at one time point postmortem [14]. Also, in a recent publication in Lancet Infectious disease [22], a total of eight COVID-19 patients who died from the disease were undergone postmortem histopathologic evaluation of tissue samples obtained by cryobiopsy within $30 \mathrm{~min}$ of death. The authors reported significant fibrotic pulmonary parenchymal remodeling, as characterized by fibroblast proliferation, micro-honeycombing, and airspace obliteration. Although intriguing, lung biopsies place patients at high risk of pneumothorax and are impractical. However, a recent report of transbronchial biopsy in a 61-year-old female COVID-19 patient demonstrated organizing pneumonia [23], which is a different pathology than fibrotic bands (Fig. 2). To our knowledge, no other studies have performed repeat lung biopsies for post-discharge surveillance. Most studies perform repeat HRCTs, but given the inherent radiation, there may be a role for low-dose or ultra-low-dose CTs in longitudinal follow-ups [24]. This is important to understand organizing pneumonia can develop either an active or aberrant pulmonary repair or recovery process during the evolution of COVID-19 [25]. Like any other etiology of organizing pneumonia, the imaging manifestation may include pulmonary consolidations and opacities in peri-bronchovascular or peri-lobular distribution, reticulation, bronchiectasis, interstitial or airspace nodules, interlobular septal thickening or even the reversed halo sign and the halo sign [26]. However, no large sample longitudinal follow-up of patients recovered from COVID-19 has been reported to date to provide a statistical estimate of the prevalence of different imaging manifestations, such as organizing pneumonia, fibrotic bands, reticulation, or honeycombing. Whether there is any overlap between the residual pulmonary consequences of COVID-19 pneumonia and non-specific interstitial pneumonia (NSIP) or usual interstitial pneumonia (UIP) is not known yet. In addition, there is a surprising lack of pulmonary function testing and spirometry in patients at and after discharge. Considering the findings from Mo et al.- that DLCO and TLC can be reduced despite meeting discharge criteria—residual pulmonary dysfunction is expected. Also, because blood oxygen levels were normal across all mild illness, pneumonia, and severe pneumonia groups in the study [11], it is possible that current discharge criteria are insufficient and/or inaccurate in determining pulmonary and functional status.

Chest radiographs can also be employed for the follow-up imaging of patients recovered from COVID-19; however, subtle parenchymal changes might not be detected on radiographs due to lower tissue resolution and overlapping tissues obscuring underlying pulmonary alterations. In fact, CT is a more sensitive means of evaluation of pulmonary parenchymal distortion. However, the choice of imaging modality depends on the clinical scenario and healthcare resources [27-30].

Given this scattering of data, methodology, and interpretations, long-term consequences of COVID-19 infection are difficult to predict. Indeed, expert opinions on practice guidelines and rehabilitation have been proposed [5-8, 10, 27], but all have yet to be validated in sufficiently powered longitudinal studies. Although current healthcare systems continue to see overwhelming numbers of new COVID-19 cases, more longitudinal studies are needed. The progression of COVID-19 clinically and radiologically remains uncertain $[31,32]$, but there is some evidence that consequences can persist for more than a month. Even if patients are found to be asymptomatic weeks or months after discharge, the possibility of insidious infection or persistent infectivity cannot be ruled out. In addition, residual abnormalities in pulmonary function and radiologic findings will be pertinent in longterm management of patients for many years to come. This can be even more prominent and common in patients with underlying pulmonary or extra-pulmonary diseases [33-36]. Post-discharge surveillance and monitoring will be necessary to avoid a cohort with chronic or permanent lung damage. Thus, continued surveillance of discharged COVID-19 patients with repeat pulmonary function testing, RT-PCR, and HRCT is recommended.

Funding Not applicable.

Availability of data and material Not applicable.

\section{Compliance with ethical standards}

Conflicts of interest The authors declare that they have no conflict of interest.

Ethical standards This article does not contain any studies with human participants or animals performed by any of the authors.

Inform consent Not applicable.

Code availability Not applicable.

\section{References}

1. Chakraborty C, Sharma AR, Sharma G et al (2020) SARS-CoV-2 causing pneumonia-associated respiratory disorder (COVID19): diagnostic and proposed therapeutic options. Eur Rev Med Pharmacol Sci 24(7):4016-4026. https://doi.org/10.26355/eurre v_202004_20871

2. Chen J, Qi T, Liu L et al (2020) Clinical progression of patients with COVID-19 in Shanghai, China. J Infect 80(5):e1-e6. https ://doi.org/10.1016/j.jinf.2020.03.004 
3. Yuan M, Yin W, Tao Z et al (2020) Association of radiologic findings with mortality of patients infected with 2019 novel coronavirus in Wuhan, China. PLoS One 15(3):e0230548. https://doi. org/10.1371/journal.pone.0230548

4. Chen N, Zhou M, Dong X et al (2020) Epidemiological and clinical characteristics of 99 cases of 2019 novel coronavirus pneumonia in Wuhan, China: a descriptive study. Lancet 395(10223):507513. https://doi.org/10.1016/S0140-6736(20)30211-7

5. Salehi S, Abedi A, Balakrishnan S, Gholamrezanezhad A (2020) Coronavirus disease 2019 (COVID-19, 2019-nCoV): a systematic review of imaging findings in 919 patients. AJR Am J Roentgenol. https://doi.org/10.2214/AJR.20.23034

6. Salehi S, Abedi A, Balakrishnan S, Gholamrezanezhad A (2019) Coronavirus disease 2019 imaging reporting and data system (COVID-RADS) and common lexicon: a proposal based on the imaging data of 37 studies. Eur Radiol. https://doi.org/10.1007/ s00330-020-06863-0

7. Salehi S, Reddy S, Gholamrezanezhad A (2019) Long-term pulmonary consequences of coronavirus disease 2019 (COVID-19): what we know and what to expect. J Thorac Imaging. https://doi. org/10.1097/RTI.0000000000000534

8. Jin et al (2020) A rapid advice guideline for the diagnosis and treatment of 2019 novel coronavirus (2019-nCoV) infected pneumonia (standard version). Mil Med Res 7:4. https://doi. org/10.1186/s40779-020-0233-6

9. Dou P, Zhang S, Wang C et al (2020) Serial CT features in discharged COVID-19 patients with positive RT-PCR re-test. Eur J Radiol. 127:109010. https://doi.org/10.1016/j.ejrad.2020.109010

10. Zheng Z, Yao ZX, Wu K, Zheng JH (2020) Patient follow-up after discharge after COVID-19 pneumonia: considerations for infectious control. J Med Virol. https://doi.org/10.1002/jmv.25994

11. Mo X, Jian W, Su Z et al (2020) Abnormal pulmonary function in COVID-19 patients at time of hospital discharge. Eur Respir J. https://doi.org/10.1183/13993003.01217-2020

12. Xing Y, Mo P, Xiao Y et al (2020) Post-discharge surveillance and positive virus detection in two medical staff recovered from coronavirus disease 2019 (COVID-19), China, January to February 2020. Eurosurveillance 25(10):2000191. https://doi. org/10.2807/1560-7917.ES.2020.25.10.2000191

13. Fu W, Chen Q, Wang T (2020) Three cases of re-detectable positive SARS-CoV-2 RNA in recovered COVID-19 patients with antibodies. J Med Virol. https://doi.org/10.1002/jmv.25968

14. Yao XH, He ZC, Li TY et al (2020) Pathological evidence for residual SARS-CoV-2 in pulmonary tissues of a ready-fordischarge patient. Cell Res. https://doi.org/10.1038/s4142 2-020-0318-5

15. Pan Y, Guan H, Zhou S et al (2020) Initial CT findings and temporal changes in patients with the novel coronavirus pneumonia (2019-nCoV): a study of 63 patients in Wuhan, China. Eur Radiol 30(6):3306-3309

16. Shi H, Han X, Jiang $\mathrm{N}$ et al (2020 Apr) Radiological findings from 81 patients with COVID-19 pneumonia in Wuhan, China: a descriptive study. Lancet Infect Dis 20(4):425-434. https://doi. org/10.1016/S1473-3099(20)30086-4

17. Liang T, Liu Z, Wu CC et al (2020) Evolution of CT findings in patients with mild COVID-19 pneumonia. Eur Radiol. https://doi. org/10.1007/s00330-020-06823-8

18. Ding X, Xu J, Zhou J, Long Q (2020) Chest CT findings of COVID-19 pneumonia by duration of symptoms. Eur J Radiol. 127:109009. https://doi.org/10.1016/j.ejrad.2020.109009

19. Pan F, Ye T, Sun P, et al. Time course of lung changes on chest CT during recovery from 2019 novel coronavirus (COVID-19) pneumonia. Radiology

20. Zhao W, Zhong Z et al (2020) CT scans of patients with 2019 novel coronavirus (COVID-19) pneumonia. Theranostics 10(10):4606-4613. https://doi.org/10.7150/thno.45016
21. Kooraki S, Hosseiny M, Gholamrezanezhad A (2020) Radiologic findings of coronavirus disease (COVID-19): clinical correlation is recommended. AJR Am J Roentgenol. https://doi. org/10.2214/AJR.20.23211

22. Grillo F, Barisione E, Ball L, Mastracci L, Fiocca R (2020) Lung fibrosis: an undervalued finding in COVID-19 pathological series [published online ahead of print, $2020 \mathrm{Jul} 28$ ]. Lancet Infect Dis. https://doi.org/10.1016/S1473-3099(20)30582-X

23. Pogatchnik BP, Swenson KE, Sharifi H, Bedi H, Berry GJ, Guo HH (2020) Radiology-pathology correlation in recovered COVID-19, demonstrating organizing pneumonia Pneumonia [published online ahead of print, $2020 \mathrm{Jul} 1$ 1]. Am J Respir Crit Care Med. https://doi.org/10.1164/rccm.202004-1278IM

24. Tofighi S, Najafi S, Johnston SK, Gholamrezanezhad A (2020) Low-dose CT in COVID-19 outbreak: radiation safety, image wisely, and image gently pledge. Emerg Radiol. 10:1-5. https ://doi.org/10.1007/s10140-020-01784-3

25. Pogatchnik BP, Swenson KE, Sharifi H, Bedi H, Berry GJ, Guo HH (2020) Radiology-pathology correlation in recovered COVID-19, demonstrating organizing [published online ahead of print, 2020 Jul 1]. Am J Respir Crit Care Med 202(4):598599. https://doi.org/10.1164/rccm.202004-1278IM

26. Faria IM, Zanetti G, Barreto MM et al (2015) Organizing pneumonia: chest HRCT findings. J Bras Pneumol 41(3):231-237. https://doi.org/10.1590/S1806-37132015000004544

27. Rubin GD, Ryerson CJ, Haramati LB et al (2020) The role of chest imaging in patient management during the COVID-19 pandemic: a multinational consensus statement from the fleischner society. Chest 158(1):106-116. https://doi.org/10.1016/j. chest.2020.04.003

28. Demirjian NL, Fields BKK, Gholamrezanezhad A (2020) Role of chest CT in resource-driven healthcare systems. Am J Roentgenol 215(3):W36

29. Cozzi D, Albanesi M, Cavigli E et al (2020) Chest X-ray in new coronavirus disease 2019 (COVID-19) infection: findings and correlation with clinical outcome. Radiol med 125:730-737. https://doi.org/10.1007/s11547-020-01232-9

30. Fields BKK, Demirjian NL, Gholamrezanezhad A (2020) Coronavirus disease 2019 (COVID-19) diagnostic technologies: a country-based retrospective analysis of screening and containment procedures during the first wave of the pandemic [published online ahead of print, 2020 Aug 26]. Clin Imaging 67:219-225. https://doi.org/10.1016/j.clinimag.2020.08.014

31. Sheehy LM (2020) Considerations for postacute rehabilitation for survivors of COVID-19. JMIR Public Health Surveill 6(2):e19462. https://doi.org/10.2196/19462

32. Kooraki S, Hosseiny M, Myers L, Gholamrezanezhad A (2020) Coronavirus (COVID-19) Outbreak: What the Department of Radiology Should Know. J Am Coll Radiol 17(4):447-451. https://doi.org/10.1016/j.jacr.2020.02.008

33. Hosseiny M, Kooraki S, Gholamrezanezhad A, Reddy S, Myers L (2020) Radiology perspective of coronavirus disease 2019 (COVID-19): lessons from severe acute respiratory syndrome and middle east respiratory syndrome. AJR Am J Roentgenol 214(5):1078-1082. https://doi.org/10.2214/AJR.20.22969

34. Behzad S, Aghaghazvini L, Radmard AR, Gholamrezanezhad A (2020) Extrapulmonary manifestations of COVID19: radiologic and clinical overview [published online ahead of print, 2020 May 18]. Clin Imaging 66:35-41. https://doi. org/10.1016/j.clinimag.2020.05.013

35. Katal S, Aghaghazvini L, Gholamrezanezhad A (2020) Chest-CT findings of COVID-19 in patients with pre-existing malignancies; a pictorial review [published online ahead of print, 2020 Jun 9]. Clin Imaging 67:121-129. https://doi. org/10.1016/j.clinimag.2020.06.004 
36. Salehi S, Abedi A, Radmard AR, Sorouri M, Gholamrezanezhad A (2020) Chest computed tomography manifestation of coronavirus disease 2019 (COVID-19) in patients with cardiothoracic conditions. J Thorac Imaging 35(4):W90-W96. https://doi. org/10.1097/RTI.0000000000000531
Publisher's Note Springer Nature remains neutral with regard to jurisdictional claims in published maps and institutional affiliations. 